\title{
Management of non-curative endoscopic submucosal dissection for early gastric cancer: do we have enough data to support this?
}

\author{
Hajime Isomoto, Hiroki Kurumi \\ Division of Medicine and Clinical Science, Department of Multidisciplinary Internal Medicine, Tottori University of Faculty of Medicine, Yonago \\ 683-8504, Japan \\ Correspondence to: Hajime Isomoto, MD, PhD. Division of Medicine and Clinical Science, Department of Multidisciplinary Internal Medicine, Tottori \\ University of Faculty of Medicine, 36-1 Nishicho, Yonago 683-8504, Japan. Email: hajimei2002@yahoo.co.jp. \\ Provenance: This is a Guest Editorial commissioned by Editor-in-Chief Jia-Fu Ji, MD, FACS (Peking University School of Oncology \& Beijing \\ Cancer Hospital, Department of Gastrointestinal Surgery, Beijing, China). \\ Comment on: Suzuki H, Oda I, Abe S, et al. Clinical outcomes of early gastric cancer patients after noncurative endoscopic submucosal dissection in a \\ large consecutive patient series. Gastric Cancer 2016. [Epub ahead of print].
}

Received: 13 March 2017; Accepted: 15 March 2017; Published: 28 April 2017.

doi: $10.21037 / \operatorname{tgh} .2017 .03 .15$

View this article at: http://dx.doi.org/10.21037/tgh.2017.03.15

Gastric cancer is one of the most common cancers. Early gastric cancer (EGC) detection is critical for its curative or even non-curative treatment. Gotoda et al. provided important information on the risks of lymph node metastasis (LNM) in a large EGC series (1). Based on their analyses, the expanded criteria for the endoscopic resection of EGC was proposed as follows mucosal cancer without ulcer findings irrespective of tumor size; mucosal cancer with ulcer findings less than $3 \mathrm{~cm}$ in diameter; and minute $(<500 \mu \mathrm{m}$ from the muscularis mucosae) submucosal invasive cancer less than $3 \mathrm{~cm}$ in size due to their nominal risks of LNM. Endoscopic submucosal dissection (ESD) has been developed worldwide as an endoluminal therapeutic technique for EGC (2). ESD allows precise histological assessment of the resected one-piece specimens in order to guide further management and to stratify a patient's risk for developing metastasis including LNM. The long-term outcome of curative EGC patients treated by ESD can be excellent; our previous study showed for the first time that the 5 -year overall survival (OS) and disease specific survival (DSS) rates of EGC patients treated by ESD were $97.1 \%$ and $100 \%$, respectively (3).

On the other hand, the outcomes of EGC patients after non-curative ESD have not been fully elucidated: in a recent issue of Gastric Cancer 2016 (PMID: 27722825), Suzuki et al. have aimed to clarify the outcomes in no less than 3,058 consecutive EGC patients who at initial onset underwent
ESD with curative intent from a single Japanese institute of representative high-volume center; National Cancer Center Hospital (4). The 569 non-curative ESD patients with a possible risk of LNM were identified to compare the outcomes between additional surgery, primarily with lymph nodes dissection and a simple follow-up. Among the 356 patients undergoing additional gastrectomy, LNM was identified in $5.1 \%$, whereas among 212 patients who underwent simple follow-up, LNM and/or distant metastases were found in $3.8 \%$ of patients. The 5 -year DSS rates were $98.8 \%$ and $96.8 \%$ for additional gastrectomy and a simple follow-up after non-curative gastric ESD. Of note, nevertheless, there was a significant difference in the 5 -year OS rates with $94.7 \%$ and $83.8 \%$, respectively. As the authors discussed, advanced age or concomitant disease might have contributed to the poor prognosis in the latter group. Indeed, the results were obtained from a retrospective assessment based on the medical records at the single major referral cancer center, but this study demonstrated the outcomes of non-curative gastric ESD patients in a large number of patients with a median followup period of more than 5 years. Prospective multi-center studies over a period of 5 years would be required to evaluate the outcomes of non-curative gastric ESD patients more precisely.

Just after this publication, Hatta et al. documented a multi-center nation-wide study to investigate long-term 
outcomes and validate the risk factors predicting recurrence after ESD (5). Of 15,785 patients who underwent ESD for EGC at 19 Japanese institutions, 1,969 patients not meeting the curative criteria were included; the patients were divided into radical surgery $(n=1,064)$ and follow-up (no additional treatment, $\mathrm{n}=905$ ) groups. OS and DSS were significantly higher in the radical surgery group than in the follow-up group $(\mathrm{P}<0.001$ and $\mathrm{P}=0.012$, respectively). However, similar to the single high volume center study aforementioned, the difference in 3-year DSS between the groups (99.4\% vs. $98.7 \%$ ) was rather small compared with the difference in 3-year OS (96.7\% vs. 84.0\%). Thus, both the large studies revealed a discrepancy between OS and DSS between the two different management groups, and concluded that a careful follow-up with no additional surgical intervention after non-curative ESD may be an acceptable option for patients at low risk of LNM, albeit further risk stratification is needed for appropriate individualized treatment strategies. In other words, additional gastrectomy should be performed particularly in non-curative gastric ESD patients with LNM risks.

According to the multi-center study conducted by Hatta et al., it was revealed that lymphatic invasion but not venous invasion was a significant independent predictor for LNM in patients with EGC who received surgical resection. Lymphatic invasion was the sole risk factor for recurrence not only in lymph nodes but also in distant organs among simple follow-up cohorts (5). It is possible that lymphatic invasion and subsequent LNM may be a primary route for recurrence in non-curative ESD in the simple followup patients. And, lymphovascular invasion was identified as an independent predictor of gastric cancer-related death in non-curative gastric ESD patients in the simple followup group (4,5). Additional surgical intervention could be determined based on a comprehensive review of the resected specimen including lymphovascular tumor emboli, tumor size, histological type and depth of invasion. However, there are currently no accurate methods to predict LNM in gastric cancer. As mentioned previously, lymphovascular emboli has been the strongest risk factor for LNM, but it is difficult to detect it using state-of art endoscopic technologies due to a paucity of biopsies prior to the endoscopic resection (6). The other imaging modalities include ultrasonography, computed tomography, magnetic resonance imaging and positron emission tomography-computed tomography for the precise detection of LNM, but their reliabilities for this purpose remains controversial (6,7). Advances in diagnostic endoscopy including magnifying endoscopy with narrow-band, blue laser and linked color imaging and the virtual chromo-endoscopy have allowed improvement in histopathological characterization of EGC via detailed imaging of tumor surface and microvascular structures $(8,9)$. However, the state-of-art techniques could not provide microscopic visualization of histology for lymphatic and microvascular emboli. Recently, laparoscopic sentinel node biopsy could be a promising method of predicting LNM in EGC (10). This approach still has technical and clinical limitations. In the retrospective study with a small number of patients, the area for lymph nodes according to the tumor and/or the lymphatic drainage of the stomach was visualized with infrared-ray electronic laparoscopy after submucosal injection of indocyanine green (ICG) around post-ESD scars. This study showed that 2 out of 20 patients had LNM which was confirmed by lymph nodes dissection without gastrectomy at a median follow-up of 61 months. As aforementioned, the EGC patients with non-curative resection can be managed with additional gastrectomy with lymph nodes dissection. Recent issues are on whether laparoscopic lymph node dissection without gastrectomy would be acceptable in non-curative gastric ESD (10), but this approach requires confirmation via further larger prospective studies.

Suzuki et al. suggested in the discussion section that a simple follow-up might be acceptable management option instead of additional gastrectomy in such non-curative ESD patients with lower survival rates as elderly patients and/ or severe concomitant diseases (4). According to Hatta et al. non-gastric cancer deaths from other causes were seen in $9.1 \%$ of the patients in the radical surgery group, whereas they were seen in no less than $29 \%$ in the followup group (5). One possible reason is that the patients in the follow-up patients were significantly older than those in the additional gastrectomy. However, both studies did not investigate life-threating concomitant diseases in detail or the causes of non-gastric cancer deaths in the non-curative ESD setting $(4,5)$. Prognostic predictors of the healthy status can be useful for decision making in cases of the non-curative gastric ESD. According to Suzuki et al., there was a significant difference in the frequencies of Charlson comorbidity index $>2$ between the additional gastrectomy and simple follow-up group (4). Previous studies, however, have shown that solid evaluation of long-term prognosis of such patients is difficult using the Charlson comorbidity index, American Society of Anesthesiologist scores and performance status $(11,12)$. There is currently no appropriate prognostic predictor for the healthy status 
of non-curative gastric ESD patients. It is not yet clear whether patients with both EGC and comorbidities are feasible candidates for ESD and whether they would consequently be able to achieve any survival benefits.

Currently, life span is becoming longer worldwide, and particularly in Japan, the mean life time is now over 80 years. Those aged more than 75 years accounted for $12.3 \%$ of the Japanese population as of 2013, and this is expected to reach $27 \%$ by 2060 (13). More importantly, the super-elderly population (aged more than 85 years) increased from 1.4 to 4.8 million during the last two decades (14). As our aging society advances, super elderly people with gastric cancer have increased annually. Opportunities to perform ESD for EGC in super elderly patients have surely increased (14). Compared to surgical intervention, ESD is less invasive, and it improves their quality of life preserving gastric function. With improvements in the devices and procedural technique, ESD itself in the patients with comorbidities such as cardiopulmonary diseases and renal disease and liver cirrhosis has been uneventfully performed in most cases, but there are still clinical problems of ESD in EGC patients with comorbid conditions (15). Several studies reported that EGC progresses to advanced gastric cancer and death within 4 or 5 years in patients who were left untreated for EGC (16). On the other hand, a retrospective analysis with the small number of patients have revealed that there is a substantially higher frequency of death in EGC patients with comorbidities than those without comorbidities in elderly patients who received ESD; that is, that the causes of death were mostly related to their comorbidities in elderly patients. The biggest cause of death in super elderly patients was a procedure-related pneumonia (14). A recent meta-analysis has shown the elderly patients had a higher procedure-related pneumonia rate compared to non-elderly patients (17).

Nowadays, ESD is an effective and safe procedure in elderly EGC patients for curative purpose. Additional gastrectomy should be performed particularly in noncurative gastric ESD patients with lymphovascular invasion even in the elderly cohort without comorbidities $(4,5)$. Nevertheless, we have not had enough data to support the real feasibility of gastric ESD linked to the survival benefits for elderly patients with comorbidities. In particular, indications of ESD should be carefully considered in each super elderly case of EGC. Suzuki $e t a l$. paved the way for us to design larger multi-center studies on risk stratification and management of non-curative resection after ESD for EGC in various personalized fashions (4). An unveiling limitation of ESD for EGC treatment is inaccuracy in
LNM (6). On the other hand some patients who underwent surgical operation after non-curative ESD were diagnosed as mucosal cancer without LNM on the final pathology. Ultimately, ESD is a curative treatment modality only if EGC cannot have LNM. Treatment strategy for EGC is ever-changing, yesterday-today-tomorrow, beyond the standard endoscopic mucosal resection via conventional ESD.

\section{Acknowledgements}

None.

\section{Footnote}

Conflicts of Interest: The authors have no conflicts of interest to declare.

\section{References}

1. Gotoda T, Yanagisawa A, Sasako M, et al. Incidence of lymph node metastasis from early gastric cancer: estimation with a large number of cases at two large centers. Gastric Cancer 2000;3:219-25.

2. Ono H, Kondo H, Gotoda T, et al. Endoscopic mucosal resection for treatment of early gastric cancer. Gut 2001;48:225-9.

3. Isomoto H, Shikuwa S, Yamaguchi N, et al. Endoscopic submucosal dissection for early gastric cancer: a large-scale feasibility study. Gut 2009;58:331-6.

4. Suzuki H, Oda I, Abe S, et al. Clinical outcomes of early gastric cancer patients after noncurative endoscopic submucosal dissection in a large consecutive patient series. Gastric Cancer 2016. [Epub ahead of print].

5. Hatta W, Gotoda T, Oyama T, et al. Is radical surgery necessary in all patients who do not meet the curative criteria for endoscopic submucosal dissection in early gastric cancer? A multi-center retrospective study in Japan. J Gastroenterol 2017;52:175-84.

6. Shin N, Jeon TY, Kim GH, et al. Unveiling lymph node metastasis in early gastric cancer. World J Gastroenterol 2014;20:5389-95.

7. Shim CN, Lee SK. Endoscopic submucosal dissection for undifferentiated-type early gastric cancer: do we have enough data to support this? World J Gastroenterol 2014;20:3938-49.

8. Kim MY, Cho JH, Cho JY. Ever-changing endoscopic treatment for early gastric cancer: yesterday-today- 
tomorrow. World J Gastroenterol 2014;20:13273-83.

9. Fukuda H, Miura Y, Hayashi, et al. Linked color imaging technology facilitates early detection of flat gastric cancers. Clin J Gastroenterol 2015;8:385-9.

10. Abe N, Takeuchi H, Ohki A, et al. Long-term outcomes of combination of endoscopic submucosal dissection and laparoscopic lymph node dissection without gastrectomy for early gastric cancer patients who have a potential risk of lymph node metastasis. Gastrointest Endosc 2011;74:792-7.

11. Kim ER, Lee H, Min BH, et al. Effect of rescue surgery after non-curative endoscopic resection of early gastric cancer. Br J Surg 2015;102:1394-401.

12. Kusano C, Iwasaki M, Kaltenbach T, et al. Should elderly patients undergo additional surgery after noncurative endoscopic resection for early gastric cancer? Long-term comparative outcomes. Am J Gastroenterol 2011;106:1064-9.

doi: $10.21037 / \operatorname{tgh} .2017 .03 .15$

Cite this article as: Isomoto $\mathrm{H}$, Kurumi H. Management of non-curative endoscopic submucosal dissection for early gastric cancer: do we have enough data to support this? Transl Gastroenterol Hepatol 2017;2:35.
13. Arai H, Ouchi $\mathrm{Y}$, Toba K, et al. Japan as the front-runner of super-aged societies: Perspectives from medicine and medical care in Japan. Geriatr Gerontol Int 2015;15:673-87.

14. Yoshifuku Y, Oka S, Tanaka S, et al. Long-term prognosis after endoscopic submucosal dissection for early gastric cancer in super-elderly patients. Surg Endosc 2016;30:4321-9.

15. Nishida T, Kato M, Yoshio T, et al. Endoscopic submucosal dissection in early gastric cancer in elderly patients and comorbid conditions. World J Gastrointest Endosc 2015;7:524-31.

16. Etoh T, Katai H, Fukagawa T, et al. Treatment of early gastric cancer in the elderly patient: results of EMR and gastrectomy at a national referral center in Japan. Gastrointest Endosc 2005;62:868-71.

17. Lin JP, Zhang YP, Xue M, et al. Endoscopic submucosal dissection for early gastric cancer in elderly patients: a meta-analysis. World J Surg Oncol 2015;13:293. 